\title{
NONLINEAR COUPLED FRACTIONAL ORDER SYSTEMS WITH INTEGRO-MULTISTRIP-MULTIPOINT BOUNDARY CONDITIONS
}

\author{
BASHIR AHMAD ${ }^{1, *}$, AHMED ALSAEDI ${ }^{2}$, SOTIRIS K. NTOUYAS $^{1,2}$ \\ ${ }^{1}$ Nonlinear Analysis and Applied Mathematics (NAAM)-Research Group, Department of Mathematics, \\ Faculty of Science, King Abdulaziz University, P.O. Box 80203, Jeddah 21589, Saudi Arabia \\ ${ }^{2}$ Department of Mathematics, University of Ioannina, 45110 Ioannina, Greece \\ *Corresponding author: bashirahmad_qau@yahoo.com
}

\begin{abstract}
We study the existence and uniqueness of solutions for a nonlinear system of coupled fractional differential equations equipped with nonlocal coupled integro-multistrip-multipoint boundary conditions. Our results are new in the sense that the given boundary conditions connect the values of the known functions over the given domain with the ones described on different sub-segments and different nonlocal positions within the given domain. We make use of Banach contraction mapping principle, Leray-Schauder alternative and Krasnoselskii fixed point theorem to prove the desired results for the problem at hand. An example illustrating the existence and uniqueness result is also presented.
\end{abstract}

\section{INTRODUCTION}

Fractional calculus is found to be more practical and effective than the classical calculus in the mathematical modeling of several real world phenomena. The topic of fractional differential equations has evolved as an important and significant area of investigation in view of its numerous applications in viscoelasticity, electroanalytical chemistry, and many physical problems [1]- [4]. In recent years, many works have been devoted to the study of the mathematical aspects of fractional order differential equations. Many advanced

Received 2019-03-22; accepted 2019-04-10; published 2019-11-01.

2010 Mathematics Subject Classification. 26A33, 34B15.

Key words and phrases. boundary value problem; fractional derivative; fractional integral; fixed point theorem.

(C)2019 Authors retain the copyrights of their papers, and all open access articles are distributed under the terms of the Creative Commons Attribution License. 
and efficient methods have been applied to develop the existence theory for fractional differential equations. One of the powerful tools for developing the existence crireria for solutions to such equations is based on the fixed point theory. Many authors applied fixed point theorems to establish the existence theory for nonlinear fractional differential equations; for example, see [5]- [13] and the references cited therein.

On the other hand, the coupled systems of nonlinear fractional differential equations also received considerable attention. Such systems appear in various disciplines of applied nature, for instance, see [14, 15]. The tools of the fixed point theory also played a key role in developing the existence theory for the coupled systems of fractional differential equations [18]- [20].

In [21], the authors investigated a coupled system of nonlinear fractional differential equations:

$$
\left\{\begin{array}{l}
{ }^{c} D^{\alpha} x(t)=f\left(t, x(t), y(t), D^{\gamma} y(t)\right), t \in[0, T], 1<\alpha \leq 2,0<\gamma<1, \\
{ }^{c} D^{\beta} y(t)=g\left(t, x(t), D^{\delta} x(t), y(t)\right), t \in[0, T], 1<\beta \leq 2,0<\delta<1,
\end{array}\right.
$$

equipped with coupled nonlocal and integral boundary conditions of the form:

$$
\left\{\begin{array}{l}
x(0)=h(y), \quad \int_{0}^{T} y(s) d s=\mu_{1} x(\eta), \\
y(0)=\phi(x), \quad \int_{0}^{T} x(s) d s=\mu_{2} y(\xi), \quad \eta, \xi \in(0, T),
\end{array}\right.
$$

where ${ }^{c} D^{i}$ denote the Caputo fractional derivatives of order $i, i=\alpha, \beta, \gamma, \delta, f, g:[0, T] \times \mathbb{R} \times \mathbb{R} \times \mathbb{R} \rightarrow \mathbb{R}$, $h, \phi: C([0, T], \mathbb{R}) \rightarrow \mathbb{R}$ are given continuous functions, and $\mu_{1}, \mu_{2}$ are real constants.

In [22], the existence of solutions for the following boundary value problem of coupled system of nonlinear fractional differential equations was discussed:

$$
\begin{aligned}
& \left\{\begin{array}{l}
{ }^{c} D^{q} x(t)=f\left(t, x(t), y(t),{ }^{c} D^{\sigma_{1}} y(t)\right), 1<q \leq 2,0<\sigma_{1}<1, t \in[0,1], \\
{ }^{c} D^{p} y(t)=g\left(t, x(t),{ }^{c} D^{\sigma_{2}} x(t), y(t)\right), 1<p \leq 2,0<\sigma_{2}<1, t \in[0,1],
\end{array}\right. \\
& \left\{\begin{array}{l}
x(0)=\psi_{1}(y), x(1)=a_{1} \int_{0}^{\xi} y(s) d s+b_{1} \sum_{i=1}^{m-2} \alpha_{i} y\left(\eta_{i}\right), \\
y(0)=\psi_{2}(x), y(1)=a_{2} \int_{0}^{\xi} x(s) d s+b_{2} \sum_{i=1}^{m-2} \beta_{i} x\left(\eta_{i}\right), \\
0<\xi<\eta_{1}<\eta_{2}<\cdots<\eta_{m-2}<1, m \geq 3
\end{array}\right.
\end{aligned}
$$

where ${ }^{c} D^{j}\left(j=p, q, \sigma_{1}, \sigma_{2}\right)$ denote the Caputo fractional derivative of order $j, f, g:[0,1] \times \mathbb{R} \times \mathbb{R} \times$ $\mathbb{R} \rightarrow \mathbb{R}, \psi_{1}, \psi_{2}: C([0,1], \mathbb{R}) \rightarrow \mathbb{R}$ are given appropriate functions, $a_{1}, a_{2}, b_{1}$ and $b_{2}$ are real constants and $\alpha_{i}, \beta_{i}, i=1,2, \ldots, m-2$, are positive real constants.

In this paper, we are concerned with existence of solutions for a nonlinear system of coupled fractional differential equations:

$$
\begin{aligned}
& D^{\sigma} x(t)=f(t, x(t), y(t)), \quad n-1<\sigma<n, n \geq 3, t \in J:=[0,1], \\
& D^{\phi} x(t)=g(t, x(t), y(t)), \quad m-1<\phi<m, m \geq 3, t \in J:=[0,1],
\end{aligned}
$$


subject to integro-multistrip-multipoint boundary conditions:

$$
\begin{aligned}
& x^{(\widehat{i})}(0)=0, \widehat{i}=0,1,2, \ldots, n-2, \\
& \int_{0}^{1} x(s) d s=\sum_{i=2}^{p} \beta_{i-1} \int_{\eta_{i-1}}^{\eta_{i}} y(s) d s+\sum_{j=1}^{q} \gamma_{j} y\left(\rho_{j}\right), \beta_{i-1}>0, \gamma_{j}>0, \\
& y^{(\widehat{j})}(0)=0, \widehat{j}=0,1,2, \ldots, m-2, \\
& \int_{0}^{1} y(s) d s=\sum_{i=2}^{\mu} \beta_{i-1}^{\prime} \int_{\theta_{i-1}}^{\theta_{i}} x(s) d s+\sum_{j=1}^{\lambda} \gamma_{j}^{\prime} x\left(\zeta_{j}\right), \beta_{i-1}^{\prime}>0, \gamma_{j}^{\prime}>0,
\end{aligned}
$$

where $D^{\sigma}, D^{\phi}$ are the standard Riemann-Liouville fractional derivatives of order $\sigma$ and $\phi$ respectively, $f, g$ : $J \times \mathbb{R} \times \mathbb{R} \rightarrow \mathbb{R}$ are continuous functions and $0<\eta_{1}<\eta_{2}<\ldots<\eta_{p}<\rho_{1}<\rho_{2}<\ldots<\rho_{q}<1$, $0<\theta_{1}<\theta_{2}<\ldots<\theta_{\mu}<\zeta_{1}<\zeta_{2}<\ldots<\zeta_{\lambda}<1$ with $p, q, \mu, \lambda \in \mathbb{N}$.

The rest of the paper is organized as follows. In Section 2, we recall some basic definitions of fractional calculus and present an auxiliary lemma, which plays a pivotal role in obtaining the main results presented in Section 3. We also discuss an example for illustration of the existence-uniqueness result.

\section{Preliminaries}

First of all, we recall some basic definitions of fractional calculus [2].

Definition 2.1. The fractional integral of order $r$ with the lower limit zero for a function $f$ is defined as

$$
I^{r} f(t)=\frac{1}{\Gamma(r)} \int_{0}^{t} \frac{f(s)}{(t-s)^{1-r}} d s, \quad t>0, \quad r>0,
$$

provided the right hand-side is point-wise defined on $[0, \infty)$, where $\Gamma(\cdot)$ is the gamma function, which is defined by $\Gamma(r)=\int_{0}^{\infty} t^{r-1} e^{-t} d t$.

Definition 2.2. The Riemann-Liouville fractional derivative of order $r>0, n-1<r<n, n \in \mathbb{N}$, is defined as

$$
D_{0+}^{r} f(t)=\frac{1}{\Gamma(n-r)}\left(\frac{d}{d t}\right)^{n} \int_{0}^{t}(t-s)^{n-r-1} f(s) d s,
$$

where the function $f$ has absolutely continuous derivatives upto order $(n-1)$.

The following lemma is of great importance in the proof of our main results.

Lemma 2.1. Let $h, k \in C(J, \mathbb{R})$ and

$$
\Omega=\frac{1}{\sigma \phi}-\Lambda_{1} \Lambda_{2} \neq 0
$$

where

$$
\Lambda_{1}=\frac{1}{\phi} \sum_{i=2}^{p} \beta_{i-1}\left(\eta_{i}^{\phi}-\eta_{i-1}^{\phi}\right)+\sum_{j=1}^{q} \gamma_{j} \rho_{j}^{\phi-1}
$$




$$
\Lambda_{2}=\frac{1}{\sigma} \sum_{i=2}^{\mu} \beta_{i-1}^{\prime}\left(\theta_{i}^{\sigma}-\theta_{i-1}^{\sigma}\right)+\sum_{j=1}^{\lambda} \gamma_{j}^{\prime} \zeta_{j}^{\sigma-1}
$$

Then the solution of the linear fractional differential system

$$
\begin{aligned}
& D^{\sigma} x(t)=h(t), \quad n-1<\sigma<n, t \in J:=[0,1], \\
& D^{\phi} y(t)=k(t), \quad m-1<\phi<m, t \in J:=[0,1],
\end{aligned}
$$

supplemented with the boundary conditions (1.2) is equivalent to the system of integral equations

$$
\begin{aligned}
x(t)= & \frac{1}{\Gamma(\sigma)} \int_{0}^{t}(t-s)^{\sigma-1} h(s) d s+\frac{t^{\sigma-1}}{\Omega}\left\{\frac { 1 } { \phi } \left(\sum_{i=2}^{p} \beta_{i-1} \frac{1}{\Gamma(\phi)} \int_{\eta_{i-1}}^{\eta_{i}} \int_{0}^{s}(s-\tau)^{\phi-1} k(s) d \tau d s\right.\right. \\
& \left.+\sum_{j=1}^{q} \gamma_{j} \frac{1}{\Gamma(\phi)} \int_{0}^{\rho_{j}}\left(\rho_{j}-s\right)^{\phi-1} k(s) d s-\frac{1}{\Gamma(\sigma)} \int_{0}^{1} \int_{0}^{t}(t-s)^{\sigma-1} h(s) d s d t\right) \\
& +\Lambda_{1}\left(\sum_{i=2}^{\mu} \beta_{i-1}^{\prime} \frac{1}{\Gamma(\sigma)} \int_{\theta_{i-1}}^{\theta_{i}} \int_{0}^{s}(s-\tau)^{\sigma-1} h(\tau) d \tau d s\right. \\
& \left.\left.+\sum_{j=1}^{\lambda} \gamma_{j}^{\prime} \frac{1}{\Gamma(\sigma)} \int_{0}^{\zeta_{j}}\left(\zeta_{j}-s\right)^{\sigma-1} h(s) d s-\frac{1}{\Gamma(\phi)} \int_{0}^{1} \int_{0}^{t}(t-s)^{\phi-1} k(s) d s d t\right)\right\}
\end{aligned}
$$

and

$$
\begin{aligned}
y(t)= & \frac{1}{\Gamma(\phi)} \int_{0}^{t}(t-s)^{\phi-1} k(s) d s+\frac{t^{\phi-1}}{\Omega}\left\{\frac { 1 } { \sigma } \left(\sum_{i=2}^{\mu} \beta_{i-1}^{\prime} \frac{1}{\Gamma(\sigma)} \int_{\theta_{i-1}}^{\theta_{i}} \int_{0}^{s}(s-\tau)^{\sigma-1} h(s) d \tau d s\right.\right. \\
& \left.+\sum_{j=1}^{\lambda} \gamma_{j}^{\prime} \frac{1}{\Gamma(\sigma)} \int_{0}^{\zeta_{j}}\left(\zeta_{j}-s\right)^{\sigma-1} h(s) d s-\frac{1}{\Gamma(\phi)} \int_{0}^{1} \int_{0}^{t}(t-s)^{\phi-1} h(s) d s d t\right) \\
& +\Lambda_{2}\left(\sum_{i=2}^{p} \beta_{i-1} \frac{1}{\Gamma(\phi)} \int_{\eta_{i-1}}^{\eta_{i}} \int_{0}^{s}(s-\tau)^{\phi-1} k(\tau) d \tau d s\right. \\
& \left.\left.+\sum_{j=1}^{q} \gamma_{j} \frac{1}{\Gamma(\phi)} \int_{0}^{\rho_{j}}\left(\rho_{j}-s\right)^{\phi-1} k(s) d s-\frac{1}{\Gamma(\sigma)} \int_{0}^{1} \int_{0}^{t}(t-s)^{\sigma-1} h(s) d s d t\right)\right\}
\end{aligned}
$$

Proof. As argued in [2], the general solution of the equations $D^{\sigma} x(t)=h(t), n-1<\sigma<n$ and $D^{\phi} y(t)=$ $k(t), m-1<\phi<m$, can be written as

$$
\begin{aligned}
& x(t)=b_{1} t^{\sigma-1}+b_{2} t^{\sigma-2}+\cdots+b_{n} t^{\sigma-n}+\frac{1}{\Gamma(\sigma)} \int_{0}^{t}(t-s)^{\sigma-1} h(s) d s, \\
& y(t)=d_{1} t^{\phi-1}+d_{2} t^{\phi-2}+\cdots+d_{m} t^{\phi-m}+\frac{1}{\Gamma(\phi)} \int_{0}^{t}(t-s)^{\phi-1} k(s) d s,
\end{aligned}
$$

where $b_{i}, i=1,2, \ldots, n$, and $d_{j}, j=1, \ldots, m$ are arbitrary constants. Using the conditions $x^{(\widehat{i})}(0)=$ $0, \widehat{i}=0,1,2, \ldots, n-2$, and $y^{(\widehat{j})}(0)=0, \widehat{j}=0,1,2, \ldots, m-2$, we find that $b_{2}=b_{3}=\cdots=b_{n}=0$ and $d_{1}=d_{2}=\ldots=d_{m}=0$. Thus $(2.4)$ and (2.5) become

$$
\begin{aligned}
& x(t)=b_{1} t^{\sigma-1}+\frac{1}{\Gamma(\sigma)} \int_{0}^{t}(t-s)^{\sigma-1} g(s) d s, \\
& y(t)=d_{1} t^{\phi-1}+\frac{1}{\Gamma(\phi)} \int_{0}^{t}(t-s)^{\phi-1} g(s) d s .
\end{aligned}
$$


Using the conditions $\int_{0}^{1} x(s) d s=\sum_{i=2}^{p} \beta_{i-1} \int_{\eta_{i-1}}^{\eta_{i}} y(s) d s+\sum_{j=1}^{q} \gamma_{j} y\left(\rho_{j}\right)$ and $\int_{0}^{1} y(s) d s=\sum_{i=2}^{\mu} \beta_{i-1}^{\prime} \int_{\theta_{i-1}}^{\theta_{i}} x(s) d s+\sum_{j=1}^{\lambda} \gamma_{j}^{\prime} x\left(\zeta_{j}\right)$ in (2.6) and (2.7), we get

$$
\begin{aligned}
\frac{1}{\sigma} b_{1}-\Lambda_{1} d_{1}= & \sum_{i=2}^{p} \beta_{i-1} \frac{1}{\Gamma(\phi)} \int_{\eta_{i-1}}^{\eta_{i}} \int_{0}^{s}(s-\tau)^{\phi-1} k(\tau) d \tau d s \\
& +\sum_{j=1}^{q} \gamma_{j} \frac{1}{\Gamma(\phi)} \int_{0}^{\rho_{j}}\left(\rho_{j}-s\right)^{\phi-1} k(s) d s-\frac{1}{\Gamma(\sigma)} \int_{0}^{1} \int_{0}^{t}(t-s)^{\sigma-1} h(s) d s d t, \\
-\Lambda_{2} b_{1}+\frac{1}{\phi} d_{1}= & \sum_{i=2}^{\mu} \beta_{i-1}^{\prime} \frac{1}{\Gamma(\sigma)} \int_{\theta_{i-1}}^{\theta_{i}} \int_{0}^{s}(s-\tau)^{\sigma-1} h(\tau) d \tau d s \\
& +\sum_{j=1}^{\lambda} \gamma_{j}^{\prime} \frac{1}{\Gamma(\sigma)} \int_{0}^{\zeta_{j}}\left(\zeta_{j}-s\right)^{\sigma-1} h(s) d s-\frac{1}{\Gamma(\phi)} \int_{0}^{1} \int_{0}^{t}(t-s)^{\phi-1} k(s) d s d t .
\end{aligned}
$$

Solving the above system for $b_{1}$ and $d_{1}$, we find that

$$
\begin{aligned}
b_{1}= & \frac{1}{\Omega}\left(\frac { 1 } { \phi } \left[\sum_{i=2}^{p} \beta_{i-1} \frac{1}{\Gamma(\phi)} \int_{\eta_{i-1}}^{\eta_{i}} \int_{0}^{s}(s-\tau)^{\phi-1} k(\tau) d \tau d s\right.\right. \\
& \left.+\sum_{j=1}^{q} \gamma_{j} \frac{1}{\Gamma(\phi)} \int_{0}^{\rho_{j}}\left(\rho_{j}-s\right)^{\phi-1} k(s) d s-\frac{1}{\Gamma(\sigma)} \int_{0}^{1} \int_{0}^{t}(t-s)^{\sigma-1} h(s) d s d t\right] \\
& +\Lambda_{1}\left[\sum_{i=2}^{\mu} \beta_{i-1}^{\prime} \frac{1}{\Gamma(\sigma)} \int_{\theta_{i-1}}^{\theta_{i}} \int_{0}^{s}(s-\tau)^{\sigma-1} h(\tau) d \tau d s\right. \\
& \left.\left.+\sum_{j=1}^{\lambda} \gamma_{j}^{\prime} \frac{1}{\Gamma(\sigma)} \int_{0}^{\zeta_{j}}\left(\zeta_{j}-s\right)^{\sigma-1} h(s) d s-\frac{1}{\Gamma(\phi)} \int_{0}^{1} \int_{0}^{t}(t-s)^{\phi-1} k(s) d s d t\right]\right),
\end{aligned}
$$

and

$$
\begin{aligned}
d_{1}= & \frac{1}{\Omega}\left(\frac { 1 } { \sigma } \left[\sum_{i=2}^{\mu} \beta_{i-1}^{\prime} \frac{1}{\Gamma(\sigma)} \int_{\theta_{i-1}}^{\theta_{i}} \int_{0}^{s}(s-\tau)^{\sigma-1} h(\tau) d \tau d s\right.\right. \\
& \left.+\sum_{j=1}^{\lambda} \gamma_{j}^{\prime} \frac{1}{\Gamma(\sigma)} \int_{0}^{\zeta_{j}}\left(\zeta_{j}-s\right)^{\sigma-1} h(s) d s-\frac{1}{\Gamma(\phi)} \int_{0}^{1} \int_{0}^{t}(t-s)^{\phi-1} k(s) d s d t\right] \\
& +\Lambda_{2}\left[\sum_{i=2}^{p} \beta_{i-1} \frac{1}{\Gamma(\phi)} \int_{\eta_{i-1}}^{\eta_{i}} \int_{0}^{s}(s-\tau)^{\phi-1} k(\tau) d \tau d s\right. \\
& \left.\left.+\sum_{j=1}^{q} \gamma_{j} \frac{1}{\Gamma(\phi)} \int_{0}^{\rho_{j}}\left(\rho_{j}-s\right)^{\phi-1} k(s) d s-\frac{1}{\Gamma(\sigma)} \int_{0}^{1} \int_{0}^{t}(t-s)^{\sigma-1} h(s) d s d t\right]\right) .
\end{aligned}
$$

Inserting the above values of $b_{1}$ and $d_{1}$ in (2.6) and (2.7) leads to the solutions (2.2) and (2.3). The converse follows by direct computation. The proof is completed.

\section{Main Results}

Let us introduce the space $X=\{x(t) \mid x(t) \in C([0,1], \mathbb{R})\}$ endowed with the norm $\|x\|=\sup \{|x(t)|, t \in$ $[0,1]\}$. Obviously $(X,\|\cdot\|)$ is a Banach space. Then the product space $(X \times X,\|(x, y)\|)$ is also a Banach space equipped with norm $\|(x, y)\|=\|x\|+\|y\|$. 
In view of Lemma 2.1, we define an operator $T: X \times X \rightarrow X \times X$ by

$$
T(x, y)(t)=\left(\begin{array}{c}
T_{1}(x, y)(t) \\
T_{2}(x, y)(t)
\end{array}\right)
$$

where

$$
\begin{aligned}
T_{1}(x, y)(t)= & \frac{1}{\Gamma(\sigma)} \int_{0}^{t}(t-s)^{\sigma-1} f(s, x(s), y(s)) d s \\
& +\frac{t^{\sigma-1}}{\Omega}\left\{\frac { 1 } { \phi } \left(\sum_{i=2}^{p} \beta_{i-1} \frac{1}{\Gamma(\phi)} \int_{\eta_{i-1}}^{\eta_{i}} \int_{0}^{s}(s-\tau)^{\phi-1} g(s, x(s), y(s)) d \tau d s\right.\right. \\
& +\sum_{j=1}^{q} \gamma_{j} \frac{1}{\Gamma(\phi)} \int_{0}^{\rho_{j}}\left(\rho_{j}-s\right)^{\phi-1} g(s, x(s), y(s)) d s \\
& \left.-\frac{1}{\Gamma(\sigma)} \int_{0}^{1} \int_{0}^{t}(t-s)^{\sigma-1} f(s, x(s), y(s)) d s d t\right) \\
& +\Lambda_{1}\left(\sum_{i=2}^{\mu} \beta_{i-1}^{\prime} \frac{1}{\Gamma(\sigma)} \int_{\theta_{i-1}}^{\theta_{i}} \int_{0}^{s}(s-\tau)^{\sigma-1} f(\tau, x(\tau), y(\tau)) d \tau d s\right. \\
& +\sum_{j=1}^{\lambda} \gamma_{j}^{\prime} \frac{1}{\Gamma(\sigma)} \int_{0}^{\zeta_{j}}\left(\zeta_{j}-s\right)^{\sigma-1} f(s, x(s), y(s)) d s \\
& \left.\left.-\frac{1}{\Gamma(\phi)} \int_{0}^{1} \int_{0}^{t}(t-s)^{\phi-1} g(s, x(s), y(s)) d s d t\right)\right\}
\end{aligned}
$$

and

$$
\begin{aligned}
T_{2}(x, y)(t)= & \frac{1}{\Gamma(\phi)} \int_{0}^{t}(t-s)^{\phi-1} g(s, x(s), y(s)) d s \\
& +\frac{t^{\phi-1}}{\Omega}\left\{\frac { 1 } { \sigma } \left(\sum_{i=2}^{\mu} \beta_{i-1}^{\prime} \frac{1}{\Gamma(\sigma)} \int_{\theta_{i-1}}^{\theta_{i}} \int_{0}^{s}(s-\tau)^{\sigma-1} f(\tau, x(\tau), y(\tau)) d \tau d s\right.\right. \\
& +\sum_{j=1}^{\lambda} \gamma_{j}^{\prime} \frac{1}{\Gamma(\sigma)} \int_{0}^{\zeta_{j}}\left(\zeta_{j}-s\right)^{\sigma-1} f(s, x(s), y(s)) d s \\
& \left.-\frac{1}{\Gamma(\phi)} \int_{0}^{1} \int_{0}^{t}(t-s)^{\phi-1} g(s, x(s), y(s)) d s d t\right) \\
& +\Lambda_{2}\left(\sum_{i=2}^{p} \beta_{i-1} \frac{1}{\Gamma(\phi)} \int_{\eta_{i-1}}^{\eta_{i}} \int_{0}^{s}(s-\tau)^{\phi-1} g(\tau, x(\tau), y(\tau)) d \tau d s\right. \\
& +\sum_{j=1}^{q} \gamma_{j} \frac{1}{\Gamma(\phi)} \int_{0}^{\rho_{j}}\left(\rho_{j}-s\right)^{\phi-1} g(s, x(s), y(s)) d s \\
& \left.\left.-\frac{1}{\Gamma(\sigma)} \int_{0}^{1} \int_{0}^{t}(t-s)^{\sigma-1} f(s, x(s), y(s)) d s d t\right)\right\}
\end{aligned}
$$

For the sake of computational convenience, we define

$$
\begin{aligned}
Q_{1}= & \frac{1}{\Gamma(\sigma+1)}+\frac{1}{|\Omega|}\left[\frac{1}{\phi} \frac{1}{\Gamma(\sigma+2)}+\left|\Lambda_{1}\right|\left(\sum_{i=2}^{\mu}\left|\beta_{i-1}^{\prime}\right| \frac{\theta_{i}^{\sigma+1}-\theta_{i-1}^{\sigma+1}}{\Gamma(\sigma+2)}\right.\right. \\
& \left.\left.+\sum_{j=1}^{\lambda}\left|\gamma_{j}^{\prime}\right| \frac{\zeta_{j}^{\sigma}}{\Gamma(\sigma+1)}\right)\right], \\
Q_{2}= & \frac{1}{|\Omega|}\left[\frac{1}{\phi}\left(\sum_{i=2}^{p}\left|\beta_{i-1}\right| \frac{\eta_{i}^{\phi+1}-\eta_{i-1}^{\phi+1}}{\Gamma(\phi+2)}+\sum_{j=1}^{q}\left|\gamma_{j}\right| \frac{\rho_{j}^{\phi}}{\Gamma(\phi+1)}\right)+\left|\Lambda_{1}\right| \frac{1}{\Gamma(\phi+2)}\right],
\end{aligned}
$$




$$
\begin{aligned}
Q_{3}= & \frac{1}{|\Omega|}\left[\frac{1}{\sigma}\left(\sum_{i=2}^{\mu}\left|\beta_{i-1}^{\prime}\right| \frac{\theta_{i}^{\sigma+1}-\theta_{i-1}^{\sigma+1}}{\Gamma(\sigma+2)}+\sum_{j=1}^{\lambda}\left|\gamma_{j}^{\prime}\right| \frac{\zeta_{j}^{\sigma}}{\Gamma(\sigma+1)}\right)+\left|\Lambda_{2}\right| \frac{1}{\Gamma(\sigma+2)}\right], \\
Q_{4}= & \frac{1}{\Gamma(\phi+1)}+\frac{1}{|\Omega|}\left[\frac{1}{\sigma} \frac{1}{\Gamma(\phi+2)}+\left|\Lambda_{2}\right|\left(\sum_{i=2}^{p}\left|\beta_{i-1}\right| \frac{\eta_{i}^{\phi+1}-\eta_{i-1}^{\phi+1}}{\Gamma(\phi+2)}\right.\right. \\
& \left.\left.+\sum_{j=1}^{q}\left|\gamma_{j}\right| \frac{\rho_{j}^{\phi}}{\Gamma(\phi+1)}\right)\right] .
\end{aligned}
$$

In the first result, we prove the existence and uniqueness of solutions for the system (1.1)-(1.2) via Banach contraction mapping principle.

Theorem 3.1. Assume that:

$\left(H_{1}\right): f, g:[0,1] \times \mathbb{R} \times \mathbb{R} \rightarrow \mathbb{R}$ are continuous functions and there exist positive constants $\ell_{1}$ and $\ell_{2}$ such that for all $t \in[0,1]$ and $x_{i}, y_{i} \in \mathbb{R}, i=1,2$, we have

$$
\begin{aligned}
& \left|f\left(t, x_{1}, x_{2}\right)-f\left(t, y_{1}, y_{2}\right)\right| \leq \ell_{1}\left(\left|x_{1}-y_{1}\right|+\left|x_{2}-y_{2}\right|\right), \\
& \left|g\left(t, x_{1}, x_{2}\right)-g\left(t, y_{1}, y_{2}\right)\right| \leq \ell_{2}\left(\left|x_{1}-y_{1}\right|+\left|x_{2}-y_{2}\right|\right) .
\end{aligned}
$$

If

$$
\left(Q_{1}+Q_{3}\right) \ell_{1}+\left(Q_{2}+Q_{4}\right) \ell_{2}<1
$$

where $Q_{i}, i=1,2,3,4$ are given by (3.3)-(3.6), then the system (1.1)-(1.2) has a unique solution on $[0,1]$.

Proof. Define $\sup _{t \in[0,1]} f(t, 0,0)=N_{1}<\infty$ and $\sup _{t \in[0,1]} g(t, 0,0)=N_{2}<\infty$ and $r>0$ such that

$$
r>\frac{\left(Q_{1}+Q_{3}\right) N_{1}+\left(Q_{2}+Q_{4}\right) N_{2}}{1-\left(Q_{1}+Q_{3}\right) \ell_{1}-\left(Q_{2}+Q_{4}\right) \ell_{2}} .
$$

We show that $T B_{r} \subset B_{r}$, where $B_{r}=\{(x, y) \in X \times X:\|(x, y)\| \leq r\}$.

By the assumption $\left(H_{1}\right)$, for $(u, v) \in B_{r}, t \in[0,1]$, we have

$$
\begin{aligned}
|f(t, x(t), y(t))| & \leq|f(t, x(t), y(t))-f(t, 0,0)|+|f(t, 0,0)| \\
& \leq \ell_{1}(|x(t)|+|y(t)|)+N_{1} \\
& \leq \ell_{1}(\|x\|+\|y\|)+N_{1} \leq \ell_{1} r+N_{1},
\end{aligned}
$$

and

$$
|g(t, x(t), y(t))| \leq \ell_{2}(\|x\|+\|y\|)+N_{2} \leq \ell_{2} r+N_{2}
$$

which lead to

$$
\begin{aligned}
\left|T_{1}(x, y)(t)\right| \leq & \frac{1}{\Gamma(\sigma)} \int_{0}^{t}(t-s)^{\sigma-1}\left(\ell_{1} r+N_{1}\right) d s \\
& +\frac{1}{|\Omega|}\left\{\frac { 1 } { \phi } \left(\sum_{i=2}^{p}\left|\beta_{i-1}\right| \frac{1}{\Gamma(\phi)} \int_{\eta_{i-1}}^{\eta_{i}} \int_{0}^{s}(s-\tau)^{\phi-1}\left(\ell_{2} r+N_{2}\right) d \tau d s\right.\right.
\end{aligned}
$$




$$
\begin{aligned}
& +\sum_{j=1}^{q}\left|\gamma_{j}\right| \frac{1}{\Gamma(\phi)} \int_{0}^{\rho_{j}}\left(\rho_{j}-s\right)^{\phi-1}\left(\ell_{2} r+N_{2}\right) d s \\
& \left.+\frac{1}{\Gamma(\sigma)} \int_{0}^{1} \int_{0}^{t}(t-s)^{\sigma-1}\left(\ell_{1} r+N_{1}\right) d s d t\right) \\
& +\left|\Lambda_{1}\right|\left(\sum_{i=2}^{\mu}\left|\beta_{i-1}^{\prime}\right| \frac{1}{\Gamma(\sigma)} \int_{\theta_{i-1}}^{\theta_{i}} \int_{0}^{s}(s-\tau)^{\sigma-1}\left(\ell_{1} r+N_{1}\right) d \tau d s\right. \\
& +\sum_{j=1}^{\lambda}\left|\gamma_{j}^{\prime}\right| \frac{1}{\Gamma(\sigma)} \int_{0}^{\zeta_{j}}\left(\zeta_{j}-s\right)^{\sigma-1}\left(\ell_{1} r+N_{1}\right) d s \\
& \left.\left.+\frac{1}{\Gamma(\phi)} \int_{0}^{1} \int_{0}^{t}(t-s)^{\phi-1}\left(\ell_{2} r+N_{2}\right) d s d t\right)\right\} \\
& =\left(Q_{1} \ell_{1}+Q_{2} \ell_{2}\right) r+Q_{1} N_{1}+Q_{2} N_{2} .
\end{aligned}
$$

Hence

$$
\left\|T_{1}(x, y)\right\| \leq\left(Q_{1} \ell_{1}+Q_{2} \ell_{2}\right) r+Q_{1} N_{1}+Q_{2} N_{2} .
$$

In a similar manner, one can find that

$$
\left\|T_{2}(x, y)\right\| \leq\left(Q_{3} \ell_{1}+Q_{4} \ell_{2}\right) r+Q_{3} N_{1}+Q_{4} N_{2} .
$$

Consequently, we have

$$
\|T(x, y)\| \leq\left[\left(Q_{1}+Q_{3}\right) \ell_{1}+\left(Q_{2}+Q_{4}\right) \ell_{2}\right] r+\left(Q_{1}+Q_{3}\right) N_{1}+\left(Q_{2}+Q_{4}\right) N_{2} \leq r .
$$

Now, for $\left(x_{2}, y_{2}\right),\left(x_{1}, y_{1}\right) \in X \times X$, and for any $t \in[0,1]$, we get

$$
\begin{aligned}
& \left|T_{1}\left(x_{2}, y_{2}\right)(t)-T_{1}\left(x_{1}, y_{1}\right)(t)\right| \\
\leq & \frac{1}{\Gamma(\sigma)} \int_{0}^{t}(t-s)^{\sigma-1} \ell_{1}\left(\left\|x_{2}-x_{1}\right\|+\left\|y_{2}-y_{1}\right\|\right) d s \\
& +\frac{1}{|\Omega|}\left\{\frac { 1 } { \phi } \left(\sum_{i=2}^{p}\left|\beta_{i-1}\right| \frac{1}{\Gamma(\phi)} \int_{\eta_{i-1}}^{\eta_{i}} \int_{0}^{s}(s-\tau)^{\phi-1} \ell_{2}\left(\left\|x_{2}-x_{1}\right\|+\left\|y_{2}-y_{1}\right\|\right) d \tau d s\right.\right. \\
& +\sum_{j=1}^{q}\left|\gamma_{j}\right| \frac{1}{\Gamma(\phi)} \int_{0}^{\rho_{j}}\left(\rho_{j}-s\right)^{\phi-1} \ell_{2}\left(\left\|x_{2}-x_{1}\right\|+\left\|y_{2}-y_{1}\right\|\right) d s \\
& \left.+\frac{1}{\Gamma(\sigma)} \int_{0}^{1} \int_{0}^{t}(t-s)^{\sigma-1} \ell_{1}\left(\left\|x_{2}-x_{1}\right\|+\left\|y_{2}-y_{1}\right\|\right) d s d t\right) \\
& +\left|\Lambda_{1}\right|\left(\sum_{i=2}^{\mu}\left|\beta_{i-1}^{\prime}\right| \frac{1}{\Gamma(\sigma)} \int_{\theta_{i-1}}^{\theta_{i}} \int_{0}^{s}(s-\tau)^{\sigma-1} \ell_{1}\left(\left\|x_{2}-x_{1}\right\|+\left\|y_{2}-y_{1}\right\|\right) d \tau d s\right. \\
& +\sum_{j=1}^{\lambda}\left|\gamma_{j}^{\prime}\right| \frac{1}{\Gamma(\sigma)} \int_{0}^{\zeta_{j}}\left(\zeta_{j}-s\right)^{\sigma-1} \ell_{1}\left(\left\|x_{2}-x_{1}\right\|+\left\|y_{2}-y_{1}\right\|\right) d s \\
& \left.\left.+\frac{1}{\Gamma(\phi)} \int_{0}^{1} \int_{0}^{t}(t-s)^{\phi-1} \ell_{2}\left(\left\|x_{2}-x_{1}\right\|+\left\|y_{2}-y_{1}\right\|\right) d s d t\right)\right\} \\
\leq \quad & \left(Q_{1} \ell_{1}+Q_{2} \ell_{2}\right)\left(\left\|x_{2}-x_{1}\right\|+\left\|y_{2}-y_{1}\right\|\right),
\end{aligned}
$$


which implies that

$$
\left\|T_{1}\left(x_{2}, y_{2}\right)-T_{1}\left(x_{1}, y_{1}\right)\right\| \leq\left(Q_{1} \ell_{1}+Q_{2} \ell_{2}\right)\left(\left\|x_{2}-x_{1}\right\|+\left\|y_{2}-y_{1}\right\|\right) .
$$

Similarly, we obtain

$$
\left\|T_{2}\left(x_{2}, y_{2}\right)(t)-T_{2}\left(x_{1}, y_{1}\right)\right\| \leq\left(Q_{3} \ell_{1}+Q_{4} \ell_{2}\right)\left(\left\|x_{2}-x_{1}\right\|+\left\|y_{2}-y_{1}\right\|\right) .
$$

Thus it follows from (3.7) and (3.8) that

$$
\left\|T\left(x_{2}, y_{2}\right)-T\left(x_{1}, y_{1}\right)\right\| \leq\left[\left(Q_{1}+Q_{3}\right) \ell_{1}+\left(Q_{2}+Q_{4}\right) \ell_{2}\right]\left(\left\|x_{2}-x_{1}\right\|+\left\|y_{2}-y_{1}\right\|\right)
$$

Since $\left(Q_{1}+Q_{3}\right) \ell_{1}+\left(Q_{2}+Q_{4}\right) \ell_{2}<1$, therefore, $T$ is a contraction. So, by Banach fixed point theorem, the operator $T$ has a unique fixed point, which corresponds to a unique solution of problem (1.1)-(1.2). This completes the proof.

In the following theorem, we prove the existence of solutions for the system (1.1)-(1.2) by means of Leray-Schauder alternative.

Lemma 3.1. (Leray-Schauder alternative) ([23] p. 4.) Let $F: E \rightarrow E$ be a completely continuous operator (i.e., a map that restricted to any bounded set in $E$ is compact). Let $\mathcal{E}(F)=\{x \in E: x=$ $\lambda F(x)$ for some $0<\lambda<1\}$. Then either the set $\mathcal{E}(F)$ is unbounded, or $F$ has at least one fixed point.

Theorem 3.2. Assume that:

$\left(H_{3}\right): f, g:[0,1] \times \mathbb{R} \times \mathbb{R} \rightarrow \mathbb{R}$ are continuous functions and there exist real constants $k_{i}, \gamma_{i} \geq 0, \quad(i=$ $0,1,2)$ and $k_{0}>0, \gamma_{0}>0$ such that $\forall x_{i} \in \mathbb{R}(i=1,2)$,

$$
\begin{aligned}
& \left|f\left(t, x_{1}, x_{2}\right)\right| \leq k_{0}+k_{1}\left|x_{1}\right|+k_{2}\left|x_{2}\right|, \\
& \left|g\left(t, x_{1}, x_{2}\right)\right| \leq \gamma_{0}+\gamma_{1}\left|x_{1}\right|+\gamma_{2}\left|x_{2}\right| .
\end{aligned}
$$

If

$$
\left(Q_{1}+Q_{3}\right) k_{1}+\left(Q_{2}+Q_{4}\right) \gamma_{1}<1 \text { and }\left(Q_{1}+Q_{3}\right) k_{2}+\left(Q_{2}+Q_{4}\right) \gamma_{2}<1
$$

where $M_{i}(i=1,2,3,4)$ are given by (3.3)-(3.6), then there exists at least one solution to the system (1.1)(1.2) on $[0,1]$.

Proof. First we show that the operator $T: X \times X \rightarrow X \times X$ is completely continuous. By continuity of functions $f$ and $g$, it is easy to show that the operator $T$ is continuous.

Let $\Omega \subset X \times X$ be bounded. Then there exist positive constants $L_{1}$ and $L_{2}$ such that

$$
|f(t, x(t), y(t))| \leq L_{1}, \quad|g(t, x(t), y(t))| \leq L_{2}, \quad \forall(x, y) \in \Omega .
$$


Then, for any $(x, y) \in \Omega$, we have

$$
\begin{aligned}
& \left|T_{1}(x, y)(t)\right| \\
\leq & \frac{1}{\Gamma(\sigma)} \int_{0}^{t}(t-s)^{\sigma-1} L_{1} d s+\frac{1}{|\Omega|}\left\{\frac { 1 } { \phi } \left(\sum_{i=2}^{p}\left|\beta_{i-1}\right| \frac{1}{\Gamma(\phi)} \int_{\eta_{i-1}}^{\eta_{i}} \int_{0}^{s}(s-\tau)^{\phi-1} L_{2} d \tau d s\right.\right. \\
& \left.+\sum_{j=1}^{q}\left|\gamma_{j}\right| \frac{1}{\Gamma(\phi)} \int_{0}^{\rho_{j}}\left(\rho_{j}-s\right)^{\phi-1} L_{2} d s+\frac{1}{\Gamma(\sigma)} \int_{0}^{1} \int_{0}^{t}(t-s)^{\sigma-1} L_{1} d s d t\right) \\
& +\left|\Lambda_{1}\right|\left(\sum_{i=2}^{\mu}\left|\beta_{i-1}^{\prime}\right| \frac{1}{\Gamma(\sigma)} \int_{\theta_{i-1}}^{\theta_{i}} \int_{0}^{s}(s-\tau)^{\sigma-1} L_{1} d \tau d s\right. \\
& \left.\left.+\sum_{j=1}^{\lambda}\left|\gamma_{j}^{\prime}\right| \frac{1}{\Gamma(\sigma)} \int_{0}^{\zeta_{j}}\left(\zeta_{j}-s\right)^{\sigma-1} L_{1} d s+\frac{1}{\Gamma(\phi)} \int_{0}^{1} \int_{0}^{t}(t-s)^{\phi-1} L_{2} d s d t\right)\right\} \\
\leq & Q_{1} L_{1}+Q_{2} L_{2},
\end{aligned}
$$

which implies that

$$
\left\|T_{1}(x, y)\right\| \leq Q_{1} L_{1}+Q_{2} L_{2}
$$

In a similar manner, we can get

$$
\left\|T_{2}(x, y)\right\| \leq Q_{3} L_{1}+Q_{4} L_{2}
$$

Thus, it follows from the above inequalities that the operator $T$ is uniformly bounded, since $\|T(x, y)\| \leq$ $\left(Q_{1}+Q_{3}\right) L_{1}+\left(Q_{2}+Q_{4}\right) L_{2}$.

Next, we show that $T$ is equicontinuous. Let $t_{1}, t_{2} \in[0,1]$ with $t_{1}<t_{2}$. Then we have

$$
\begin{aligned}
& \left|T_{1}\left(x\left(t_{2}\right), y\left(t_{2}\right)\right)-T_{1}\left(x\left(t_{1}\right), y\left(t_{1}\right)\right)\right| \\
\leq & L_{1}\left\{\frac{1}{\Gamma(\sigma)} \int_{0}^{t_{1}}\left[\left(t_{2}-s\right)^{\sigma-1}-\left(t_{1}-s\right)^{\sigma-1}\right] d s+\frac{1}{\Gamma(\sigma)} \int_{t_{1}}^{t_{2}}\left(t_{2}-s\right)^{\sigma-1} d s\right\} \\
& +\frac{\left|t_{2}^{\sigma-1}-t_{1}^{\sigma-1}\right|}{|\Omega|}\left\{\left[\frac{1}{\phi} \frac{1}{\Gamma(\sigma+2)}+\left|\Lambda_{1}\right|\left(\sum_{i=2}^{\mu}\left|\beta_{i-1}^{\prime}\right| \frac{\left.\left.\theta_{i}^{\sigma+1}-\theta_{i-1}^{\sigma+1}\right)\right] L_{1}}{\Gamma(\sigma+2)}\right.\right.\right. \\
& \left.+\left[\frac{1}{\phi}\left(\sum_{i=2}^{p}\left|\beta_{i-1}\right| \frac{\eta_{i}^{\phi+1}-\eta_{i-1}^{\phi+1}}{\Gamma(\phi+2)}+\sum_{j=1}^{q}\left|\gamma_{j}\right| \frac{\rho_{j}^{\phi}}{\Gamma(\phi+1)}\right)+\left|\Lambda_{1}\right| \frac{1}{\Gamma(\phi+2)}\right] L_{2}\right\} \\
\leq & \frac{L_{1}}{\Gamma(\sigma+1)}\left[2\left(t_{2}-t_{1}\right)^{\sigma}+\left|t_{2}^{\sigma}-t_{1}^{\sigma}\right|\right] \\
& +\frac{\left|t_{2}^{\sigma-1}-t_{1}^{\sigma-1}\right|}{|\Omega|}\left\{\left[\frac{1}{\phi} \frac{1}{\Gamma(\sigma+2)}+\left|\Lambda_{1}\right|\left(\sum_{i=2}^{\mu}\left|\beta_{i-1}^{\prime}\right| \frac{\left.\left.\theta_{i}^{\sigma+1}-\theta_{i-1}^{\sigma+1}\right)\right] L_{1}}{\Gamma(\sigma+2)}\right.\right.\right. \\
& \left.+\left[\frac{1}{\phi}\left(\sum_{i=2}^{p}\left|\beta_{i-1}\right| \frac{\eta_{i}^{\phi+1}-\eta_{i-1}^{\phi+1}}{\Gamma(\phi+2)}+\sum_{j=1}^{q}\left|\gamma_{j}\right| \frac{\rho_{j}^{\phi}}{\Gamma(\phi+1)}\right)+\left|\Lambda_{1}\right| \frac{1}{\Gamma(\phi+2)}\right] L_{2}\right\} .
\end{aligned}
$$

Analogously, we can obtain

$$
\left|T_{2}\left(x\left(t_{2}\right), y\left(t_{2}\right)\right)-T_{2}\left(x\left(t_{1}\right), y\left(t_{1}\right)\right)\right|
$$




$$
\begin{aligned}
\leq & \frac{L_{2}}{\Gamma(\phi+1)}\left[2\left(t_{2}-t_{1}\right)^{\phi}+\left|t_{2}-t_{1}^{\phi}\right|\right] \\
& +\frac{\left|t_{2}^{\phi-1}-t_{1}^{\phi-1}\right|}{|\Omega|}\left\{\left[\frac{1}{\sigma}\left(\sum_{i=2}^{\mu}\left|\beta_{i-1}^{\prime}\right| \frac{\theta_{i}^{\sigma+1}-\theta_{i-1}^{\sigma+1}}{\Gamma(\sigma+2)}+\sum_{j=1}^{\lambda}\left|\gamma_{j}^{\prime}\right| \frac{\zeta_{j}^{\sigma}}{\Gamma(\sigma+1)}\right)+\left|\Lambda_{2}\right| \frac{1}{\Gamma(\sigma+2)}\right] L_{1}\right. \\
& \left.+\left[\frac{1}{\sigma} \frac{1}{\Gamma(\phi+2)}+\left|\Lambda_{2}\right|\left(\sum_{i=2}^{p}\left|\beta_{i-1}\right| \frac{\eta_{i}^{\phi+1}-\eta_{i-1}^{\phi+1}}{\Gamma(\phi+2)}+\sum_{j=1}^{q}\left|\gamma_{j}\right| \frac{\rho_{j}^{\phi}}{\Gamma(\phi+1)}\right)\right] L_{2}\right\} .
\end{aligned}
$$

From the preceding inequalities, we deduce that the operator $T(x, y)$ is equicontinuous, and thus the operator $T(x, y)$ is completely continuous.

Finally, it will be verified that the set $\mathcal{E}=\{(x, y) \in X \times X \mid(x, y)=\lambda T(x, y), 0 \leq \lambda \leq 1\}$ is bounded. Let $(x, y) \in \mathcal{E}$, with $(x, y)=\lambda T(x, y)$. For any $t \in[0,1]$, we have

$$
x(t)=\lambda T_{1}(x, y)(t), \quad y(t)=\lambda T_{2}(x, y)(t) .
$$

Then

$$
\begin{aligned}
|x(t)| & \leq Q_{1}\left(k_{0}+k_{1}|x|+k_{2}|y|\right)+Q_{2}\left(\gamma_{0}+\gamma_{1}|x|+\gamma_{2}|y|\right) \\
& =Q_{1} k_{0}+Q_{2} \gamma_{0}+\left(Q_{1} k_{1}+Q_{2} \gamma_{1}\right)|x|+\left(Q_{1} k_{2}+Q_{2} \gamma_{2}\right)|y|
\end{aligned}
$$

and

$$
\begin{aligned}
|y(t)| & \leq Q_{3}\left(k_{0}+k_{1}|x|+k_{2}|y|\right)+Q_{4}\left(\gamma_{0}+\gamma_{1}|x|+\gamma_{2}|y|\right) \\
& =Q_{3} k_{0}+Q_{4} \gamma_{0}+\left(Q_{3} k_{1}+Q_{4} \gamma_{1}\right)|x|+\left(Q_{3} k_{2}+Q_{4} \gamma_{2}\right)|y|
\end{aligned}
$$

Hence we have

$$
\|x\| \leq Q_{1} k_{0}+Q_{2} \gamma_{0}+\left(Q_{1} k_{1}+Q_{2} \gamma_{1}\right)\|x\|+\left(Q_{1} k_{2}+Q_{2} \gamma_{2}\right)\|y\|
$$

and

$$
\|y\| \leq Q_{3} k_{0}+Q_{4} \gamma_{0}+\left(Q_{3} k_{1}+Q_{4} \gamma_{1}\right)\|x\|+\left(Q_{3} k_{2}+Q_{4} \gamma_{2}\right)\|y\|
$$

which imply that

$$
\begin{aligned}
\|x\|+\|y\| \leq & \left(Q_{1}+Q_{3}\right) k_{0}+\left(Q_{2}+Q_{4}\right) \gamma_{0}+\left[\left(Q_{1}+Q_{3}\right) k_{1}+\left(Q_{2}+Q_{4}\right) \gamma_{1}\right]\|x\| \\
& \left.+\left[\left(Q_{1}+Q_{3}\right) k_{2}+\left(Q_{2}+Q_{4}\right) \gamma_{2}\right)\right]\|y\| .
\end{aligned}
$$

Consequently,

$$
\|(x, y)\| \leq \frac{\left(Q_{1}+Q_{3}\right) k_{0}+\left(Q_{2}+Q_{4}\right) \gamma_{0}}{M_{0}},
$$

where $\left.M_{0}=\min \left\{1-\left[\left(Q_{1}+Q_{3}\right) k_{1}+\left(Q_{2}+Q_{4}\right) \gamma_{1}\right], 1-\left[\left(Q_{1}+Q_{3}\right) k_{2}+\left(Q_{2}+Q_{4}\right) \gamma_{2}\right)\right]\right\}$, which proves that $\mathcal{E}$ is bounded. Thus, by Lemma 3.1, the operator $T$ has at least one fixed point. Hence the system (1.1)-(1.2) has at least one solution. The proof is complete.

Our last result is based on Krasnoselskii fixed point theorem [24]. 
Lemma 3.2. (Krasnoselskii) Let $M$ be a closed, bounded, convex and nonempty subset of a Banach space $X$. Let $A, B$ be operators mapping $M$ to $X$ such that

(a) $A x+B y \in M$ where $x, y \in M$;

(b) $A$ is compact and continuous;

(c) B is a contraction mapping.

Then there exists $z \in M$ such that $z=A z+B z$.

Theorem 3.3. Assume that $f, g:[0,1] \times \mathbb{R} \rightarrow \mathbb{R}$ are continuous functions satisfying assumption $\left(H_{1}\right)$ in Theorem 3.1. In addition we suppose that there exist two positive constants $L_{1}, L_{2}$ such that for all $t \in[0,1]$ and $x_{i}, y_{i} \in \mathbb{R}, i=1,2$,

$$
\left|f\left(t, x_{1}, x_{2}\right)\right| \leq L_{1} \text { and }\left|g\left(t, x_{1}, x_{2}\right)\right| \leq L_{2} .
$$

If

$$
\frac{\ell_{1}}{\Gamma(\sigma+1)}+\frac{\ell_{2}}{\Gamma(\phi+1)}<1
$$

then the problem (1.1)-(1.2) has at least one solution on $[0,1]$.

Proof. In order to verify the hypotheses of Lemma 3.2, we decompose the operator $T$ into four operators $T_{1,1}, T_{1,2}, T_{2,1}$ and $T_{2,2}$ on $B_{\delta}=\{(x, y) \in X \times Y:\|(x, y)\| \leq \delta\}$ as follows:

$$
\begin{aligned}
T_{1,1}(x, y)(t)= & \frac{t^{\sigma-1}}{\Omega}\left\{\frac { 1 } { \phi } \left(\sum_{i=2}^{p} \beta_{i-1} \frac{1}{\Gamma(\phi)} \int_{\eta_{i-1}}^{\eta_{i}} \int_{0}^{s}(s-\tau)^{\phi-1} g(s, x(s), y(s)) d \tau d s\right.\right. \\
& +\sum_{j=1}^{q} \gamma_{j} \frac{1}{\Gamma(\phi)} \int_{0}^{\rho_{j}}\left(\rho_{j}-s\right)^{\phi-1} g(s, x(s), y(s)) d s \\
& \left.-\frac{1}{\Gamma(\sigma)} \int_{0}^{1} \int_{0}^{t}(t-s)^{\sigma-1} f(s, x(s), y(s)) d s d t\right) \\
& +\Lambda_{1}\left(\sum_{i=2}^{\mu} \beta_{i-1}^{\prime} \frac{1}{\Gamma(\sigma)} \int_{\theta_{i-1}}^{\theta_{i}} \int_{0}^{s}(s-\tau)^{\sigma-1} f(\tau, x(\tau), y(\tau)) d \tau d s\right. \\
& +\sum_{j=1}^{\lambda} \gamma_{j}^{\prime} \frac{1}{\Gamma(\sigma)} \int_{0}^{\zeta_{j}}\left(\zeta_{j}-s\right)^{\sigma-1} f(s, x(s), y(s)) d s \\
& \left.\left.-\frac{1}{\Gamma(\phi)} \int_{0}^{1} \int_{0}^{t}(t-s)^{\phi-1} g(s, x(s), y(s)) d s d t\right)\right\}, \\
T_{1,2}(x, y)(t)= & \frac{1}{\Gamma(\sigma)} \int_{0}^{t}(t-s)^{\sigma-1} f(s, x(s), y(s)) d s,
\end{aligned}
$$

and

$$
\begin{aligned}
T_{2,1}(x, y)(t)= & \frac{t^{\phi-1}}{\Omega}\left\{\frac { 1 } { \sigma } \left(\sum_{i=2}^{\mu} \beta_{i-1}^{\prime} \frac{1}{\Gamma(\sigma)} \int_{\theta_{i-1}}^{\theta_{i}} \int_{0}^{s}(s-\tau)^{\sigma-1} f(\tau, x(\tau), y(\tau)) d \tau d s\right.\right. \\
& +\sum_{j=1}^{\lambda} \gamma_{j}^{\prime} \frac{1}{\Gamma(\sigma)} \int_{0}^{\zeta_{j}}\left(\zeta_{j}-s\right)^{\sigma-1} f(s, x(s), y(s)) d s \\
& \left.-\frac{1}{\Gamma(\phi)} \int_{0}^{1} \int_{0}^{t}(t-s)^{\phi-1} g(s, x(s), y(s)) d s d t\right)
\end{aligned}
$$




$$
\begin{aligned}
& +\Lambda_{2}\left(\sum_{i=2}^{p} \beta_{i-1} \frac{1}{\Gamma(\phi)} \int_{\eta_{i-1}}^{\eta_{i}} \int_{0}^{s}(s-\tau)^{\phi-1} g(\tau, x(\tau), y(\tau)) d \tau d s\right. \\
& +\sum_{j=1}^{q} \gamma_{j} \frac{1}{\Gamma(\phi)} \int_{0}^{\rho_{j}}\left(\rho_{j}-s\right)^{\phi-1} g(s, x(s), y(s)) d s \\
& \left.\left.-\frac{1}{\Gamma(\sigma)} \int_{0}^{1} \int_{0}^{t}(t-s)^{\sigma-1} f(s, x(s), y(s)) d s d t\right)\right\}, \\
T_{2,2}(x, y)(t)= & \frac{1}{\Gamma(\phi)} \int_{0}^{t}(t-s)^{\phi-1} g(s, x(s), y(s)) d s .
\end{aligned}
$$

Notice that $T_{1}(x, y)(t)=T_{1,1}(x, y)(t)+T_{1,2}(x, y)(t)$ and $T_{2}(x, y)(t)=T_{2,1}(x, y)(t)+T_{2,2}(x, y)(t)$ on $B_{\delta}$ and that the ball $B_{\delta}$ is a closed, bounded and convex subset of the Banach space $X \times X$. Let us select $\delta \geq \max \left\{Q_{1} L_{1}+Q_{2} L_{2}, Q_{3} L_{1}+Q_{4} L_{2}\right\}$ and show that $T B_{\delta} \subset B_{\delta}$ for verifying condition (a) of Lemma 3.2. Setting $x=\left(x_{1}, x_{2}\right), y=\left(y_{1}, y_{2}\right) \in B_{\delta}$, and using condition (3.9), we obtain

$$
\begin{aligned}
& \left|T_{1,1}(x, y)(t)+T_{1,2}(x, y)(t)\right| \\
\leq & \frac{1}{\Gamma(\sigma)} \int_{0}^{t}(t-s)^{\sigma-1} L_{1} d s+\frac{1}{|\Omega|}\left\{\frac { 1 } { \phi } \left(\sum_{i=2}^{p}\left|\beta_{i-1}\right| \frac{1}{\Gamma(\phi)} \int_{\eta_{i-1}}^{\eta_{i}} \int_{0}^{s}(s-\tau)^{\phi-1} L_{2} d \tau d s\right.\right. \\
& \left.+\sum_{j=1}^{q}\left|\gamma_{j}\right| \frac{1}{\Gamma(\phi)} \int_{0}^{\rho_{j}}\left(\rho_{j}-s\right)^{\phi-1} L_{2} d s+\frac{1}{\Gamma(\sigma)} \int_{0}^{1} \int_{0}^{t}(t-s)^{\sigma-1} L_{1} d s d t\right) \\
& +\left|\Lambda_{1}\right|\left(\sum_{i=2}^{\mu}\left|\beta_{i-1}^{\prime}\right| \frac{1}{\Gamma(\sigma)} \int_{\theta_{i-1}}^{\theta_{i}} \int_{0}^{s}(s-\tau)^{\sigma-1} L_{1} d \tau d s\right. \\
& \left.\left.+\sum_{j=1}^{\lambda}\left|\gamma_{j}^{\prime}\right| \frac{1}{\Gamma(\sigma)} \int_{0}^{\zeta_{j}}\left(\zeta_{j}-s\right)^{\sigma-1} L_{1} d s+\frac{1}{\Gamma(\phi)} \int_{0}^{1} \int_{0}^{t}(t-s)^{\phi-1} L_{2} d s d t\right)\right\} \\
= & Q_{1} L_{1}+Q_{2} L_{2} \leq \delta .
\end{aligned}
$$

Likewise, we can find that

$$
\left|T_{2,1}(\hat{x}, \hat{y})(t)+T_{2,2}(\hat{x}, \hat{y})(t)\right| \leq Q_{3} L_{1}+Q_{4} L_{2} \leq \delta
$$

Clearly the above two inequalities lead to the fact that $T_{1}(x, y)+T_{2}(\hat{x}, \hat{y}) \in B_{\delta}$.

Now we establish that the operator $\left(T_{1,2}, T_{2,2}\right)$ is a contraction satisfying condition (c) of Lemma 3.2. For $\left(x_{1}, y_{1}\right),\left(x_{2}, y_{2}\right) \in B_{\delta}$, we have

$$
\begin{aligned}
& \left|T_{1,2}\left(x_{1}, y_{1}\right)(t)-T_{1,2}\left(x_{2}, y_{2}\right)(t)\right| \\
= & \frac{1}{\Gamma(\sigma)} \int_{0}^{t}(t-s)^{\sigma-1}\left|f\left(s, x_{1}(s), y_{1}(s)\right)-f\left(s, x_{2}(s), y_{2}(s)\right)\right| d s \\
\leq & \frac{\ell_{1}}{\Gamma(\sigma+1)}\left(\left\|x_{1}-y_{1}\right\|+\left\|x_{2}-y_{2}\right\|\right),
\end{aligned}
$$

and

$$
\left|T_{2,2}\left(x_{1}, y_{1}\right)(t)-T_{2,2}\left(x_{2}, y_{2}\right)(t)\right|
$$




$$
\begin{aligned}
& =\frac{1}{\Gamma(\phi)} \int_{0}^{t}(t-s)^{\phi-1}\left|g\left(s, x_{1}(s), y_{1}(s)\right)-g\left(s, x_{2}(s), y_{2}(s)\right)\right| d s \\
& \leq \frac{\ell_{2}}{\Gamma(\phi+1)}\left(\left\|x_{1}-y_{1}\right\|+\left\|x_{2}-y_{2}\right\|\right) .
\end{aligned}
$$

It follows from (3.11) and (3.12) that

$$
\begin{aligned}
& \left\|\left(T_{1,2}, T_{2,2}\right)\left(x_{1}, y_{1}\right)-\left(T_{1,2}, T_{2,2}\right)\left(x_{2}, y_{2}\right)\right\| \\
\leq & {\left[\frac{\ell_{1}}{\Gamma(\sigma+1)}+\frac{\ell_{2}}{\Gamma(\phi+1)}\right]\left(\left\|x_{1}-y_{1}\right\|+\left\|x_{2}-y_{2}\right\|\right), }
\end{aligned}
$$

which is a contraction by (3.10). Therefore, the condition (c) of Lemma 3.2 is satisfied.

Next we will show that the operator $\left(T_{1,1}, T_{2,1}\right)$ satisfies the condition (b) of Lemma 3.2. By applying the continuity of the functions $f, g$ on $[0,1] \times \mathbb{R} \times \mathbb{R}$, we can conclude that the operator $\left(T_{1,1}, T_{2,1}\right)$ is continuous. For each $(x, y) \in B_{\delta}$, we have

$$
\begin{aligned}
& \left|T_{1,1}(x, y)(t)\right| \\
\leq & \frac{1}{|\Omega|}\left\{\frac { 1 } { \phi } \left(\sum_{i=2}^{p}\left|\beta_{i-1}\right| \frac{1}{\Gamma(\phi)} \int_{\eta_{i-1}}^{\eta_{i}} \int_{0}^{s}(s-\tau)^{\phi-1} L_{2} d \tau d s\right.\right. \\
& \left.+\sum_{j=1}^{q}\left|\gamma_{j}\right| \frac{1}{\Gamma(\phi)} \int_{0}^{\rho_{j}}\left(\rho_{j}-s\right)^{\phi-1} L_{2} d s+\frac{1}{\Gamma(\sigma)} \int_{0}^{1} \int_{0}^{t}(t-s)^{\sigma-1} L_{1} d s d t\right) \\
& +\left|\Lambda_{1}\right|\left(\sum_{i=2}^{\mu}\left|\beta_{i-1}^{\prime}\right| \frac{1}{\Gamma(\sigma)} \int_{\theta_{i-1}}^{\theta_{i}} \int_{0}^{s}(s-\tau)^{\sigma-1} L_{1} d \tau d s\right. \\
& \left.\left.+\sum_{j=1}^{\lambda}\left|\gamma_{j}^{\prime}\right| \frac{1}{\Gamma(\sigma)} \int_{0}^{\zeta_{j}}\left(\zeta_{j}-s\right)^{\sigma-1} L_{1} d s+\frac{1}{\Gamma(\phi)} \int_{0}^{1} \int_{0}^{t}(t-s)^{\phi-1} L_{2} d s d t\right)\right\} \\
= & P^{*}
\end{aligned}
$$

and

$$
\begin{aligned}
& \left|T_{2,1}(x, y)(t)\right| \\
\leq & \frac{t^{\phi-1}}{|\Omega|}\left\{\frac { 1 } { \sigma } \left(\sum_{i=2}^{\mu} \beta_{i-1}^{\prime} \frac{1}{\Gamma(\sigma)} \int_{\theta_{i-1}}^{\theta_{i}} \int_{0}^{s}(s-\tau)^{\sigma-1} L_{1} d \tau d s\right.\right. \\
& \left.+\sum_{j=1}^{\lambda} \gamma_{j}^{\prime} \frac{1}{\Gamma(\sigma)} \int_{0}^{\zeta_{j}}\left(\zeta_{j}-s\right)^{\sigma-1} L_{1} d s+\frac{1}{\Gamma(\phi)} \int_{0}^{1} \int_{0}^{t}(t-s)^{\phi-1} L_{2} d s d t\right) \\
& +\Lambda_{2}\left(\sum_{i=2}^{p} \beta_{i-1} \frac{1}{\Gamma(\phi)} \int_{\eta_{i-1}}^{\eta_{i}} \int_{0}^{s}(s-\tau)^{\phi-1} L_{2} d \tau d s\right. \\
& \left.\left.+\sum_{j=1}^{q} \gamma_{j} \frac{1}{\Gamma(\phi)} \int_{0}^{\rho_{j}}\left(\rho_{j}-s\right)^{\phi-1} L_{2} d s+\frac{1}{\Gamma(\sigma)} \int_{0}^{1} \int_{0}^{t}(t-s)^{\sigma-1} L_{1} d s d t\right)\right\} \\
= & Q^{*},
\end{aligned}
$$


which lead to the fact that

$$
\left\|\left(T_{1,1}, T_{2,1}\right)(x, y)\right\| \leq P^{*}+Q^{*}
$$

Thus the set $\left(T_{1,1}, T_{2,1}\right) B_{\delta}$ is uniformly bounded. In the next step, we will show that the set $\left(T_{1,1}, T_{2,1}\right) B_{\delta}$ is equicontinuous. For $t_{1}, t_{2} \in[0,1]$ with $t_{1}<t_{2}$ and for any $(x, y) \in B_{\delta}$, we obtain

$$
\begin{aligned}
& \left|T_{1,1}(x, y)\left(t_{2}\right)-T_{1,1}(x, y)\left(t_{1}\right)\right| \\
\leq & \frac{\left|t_{2}^{\sigma-1}-t_{1}^{\sigma-1}\right|}{|\Omega|}\left\{\left[\frac{1}{\phi} \frac{1}{\Gamma(\sigma+2)}+\left|\Lambda_{1}\right|\left(\sum_{i=2}^{\mu}\left|\beta_{i-1}^{\prime}\right| \frac{\theta_{i}^{\sigma+1}-\theta_{i-1}^{\sigma+1}}{\Gamma(\sigma+2)}\right)\right] L_{1}\right. \\
& \left.+\left[\frac{1}{\phi}\left(\sum_{i=2}^{p}\left|\beta_{i-1}\right| \frac{\eta_{i}^{\phi+1}-\eta_{i-1}^{\phi+1}}{\Gamma(\phi+2)}+\sum_{j=1}^{q}\left|\gamma_{j}\right| \frac{\rho_{j}^{\phi}}{\Gamma(\phi+1)}\right)+\left|\Lambda_{1}\right| \frac{1}{\Gamma(\phi+2)}\right] L_{2}\right\} \\
\leq & +\frac{\left|t_{2}^{\sigma-1}-t_{1}^{\sigma-1}\right|}{|\Omega|}\left\{\left[\frac{1}{\phi} \frac{1}{\Gamma(\sigma+2)}+\left|\Lambda_{1}\right|\left(\sum_{i=2}^{\mu}\left|\beta_{i-1}^{\prime}\right| \frac{\theta_{i}^{\sigma+1}-\theta_{i-1}^{\sigma+1}}{\Gamma(\sigma+2)}\right)\right] L_{1}\right. \\
& \left.+\left[\frac{1}{\phi}\left(\sum_{i=2}^{p}\left|\beta_{i-1}\right| \frac{\eta_{i}^{\phi+1}-\eta_{i-1}^{\phi+1}}{\Gamma(\phi+2)}+\sum_{j=1}^{q}\left|\gamma_{j}\right| \frac{\rho_{j}^{\phi}}{\Gamma(\phi+1)}\right)+\left|\Lambda_{1}\right| \frac{1}{\Gamma(\phi+2)}\right] L_{2}\right\} .
\end{aligned}
$$

Analogously, we can obtain

$$
\begin{aligned}
& \left|T_{2,1}(x, y)\left(t_{2}\right)-T_{2,1}(x, y)\left(t_{1}\right)\right| \\
\leq & \frac{\left|t_{2}^{\phi-1}-t_{1}^{\phi-1}\right|}{|\Omega|}\left\{\left[\frac{1}{\sigma}\left(\sum_{i=2}^{\mu}\left|\beta_{i-1}^{\prime}\right| \frac{\theta_{i}^{\sigma+1}-\theta_{i-1}^{\sigma+1}}{\Gamma(\sigma+2)}+\sum_{j=1}^{\lambda}\left|\gamma_{j}^{\prime}\right| \frac{\zeta_{j}^{\sigma}}{\Gamma(\sigma+1)}\right)+\left|\Lambda_{2}\right| \frac{1}{\Gamma(\sigma+2)}\right] L_{1}\right. \\
& \left.+\left[\frac{1}{\sigma} \frac{1}{\Gamma(\phi+2)}+\left|\Lambda_{2}\right|\left(\sum_{i=2}^{p}\left|\beta_{i-1}\right| \frac{\eta_{i}^{\phi+1}-\eta_{i-1}^{\phi+1}}{\Gamma(\phi+2)}+\sum_{j=1}^{q}\left|\gamma_{j}\right| \frac{\rho_{j}^{\phi}}{\Gamma(\phi+1)}\right)\right] L_{2}\right\} .
\end{aligned}
$$

Thus $\left|\left(T_{1,1}, T_{2,1}\right)(x, y)\left(t_{2}\right)-\left(T_{1,1}, T_{2,1}\right)(x, y)\left(t_{1}\right)\right|$ tends to zero as $t_{1} \rightarrow t_{2}$ independent of $(x, y) \in B_{\delta}$. Therefore the set $\left(T_{1,1}, T_{2,1}\right) B_{\delta}$ is equicontinuous. Thus it follows by the Arzelá-Ascoli theorem that the operator $\left(T_{1,1}, T_{2,1}\right)$ is compact on $B_{\delta}$. By the conclusion of Lemma 3.2, we deduce that the problem (1.1)(1.2) has at least one solution on $[0,1]$. This completes the proof.

Example 3.1. Consider the following system of fractional boundary value problems

$$
\left\{\begin{array}{l}
{ }^{c} D^{3 / 2} x(t)=\frac{1}{4(t+2)^{2}} \frac{|x(t)|}{1+|x(t)|}+1+\frac{1}{32} \sin ^{2} y(t)+\frac{1}{\sqrt{t^{2}+1}}, t \in[0,1], \\
{ }^{c} D^{5 / 2} y(t)=\frac{1}{32 \pi} \sin (2 \pi x(t))+\frac{|y(t)|}{16(1+|y(t)|)}+\frac{1}{2}, t \in[0,1], \\
\int_{0}^{1} x(s) d s=\frac{5}{2} \int_{1 / 7}^{1 / 6} y(s) d s+4 \int_{1 / 6}^{1 / 5} y(s) d s+\frac{3}{2} y\left(\frac{1}{4}\right)+2 y\left(\frac{1}{3}\right), \\
\int_{0}^{1} y(s) d s=2 \int_{1 / 3}^{1 / 2} x(s) d s+4 \int_{1 / 2}^{2 / 3} x(s) d s+x\left(\frac{1}{3}\right)+\frac{1}{2} x\left(\frac{2}{3}\right) .
\end{array}\right.
$$

Here $\sigma=3 / 2, \phi=3 / 2, \beta_{1}=5 / 2, \beta_{2}=4, \eta_{1}=1 / 7, \eta_{2}=1 / 6, \eta_{3}=1 / 5, \gamma_{1}=3 / 2, \gamma_{2}=2, \rho_{1}=1 / 4, \rho_{2}=$ $1 / 3, \beta_{1}^{\prime}=2, \beta_{2}^{\prime}=4, \gamma_{1}^{\prime}=1, \gamma_{2}^{\prime}=1 / 2, \theta_{1}=1 / 3, \theta_{2}=1 / 2, \theta_{3}=2 / 3, \zeta_{1}=1 / 3, \zeta_{2}=2 / 3, f(t, x, y)=$ $\frac{1}{4(t+2)^{2}} \frac{|x|}{1+|x|}+1+\frac{1}{32} \sin ^{2} y$, and $g(t, x, y)=\frac{1}{32 \pi} \sin (2 \pi x)+\frac{|y|}{16(1+|y|)}+\frac{1}{2}$. With the given data, we find 
that $Q_{1} \approx 1.618712, Q_{2} \approx 0.096199, Q_{3} \approx 1.276613, Q_{4} \approx 0.199029$. Note that $\left|f\left(t, x_{1}, x_{2}\right)-f\left(t, y_{1}, y_{2}\right)\right| \leq$ $\frac{1}{16}\left|x_{1}-x_{2}\right|+\frac{1}{16}\left|y_{1}-y_{2}\right|,\left|g\left(t, x_{1}, x_{2}\right)-g\left(t, y_{1}, y_{2}\right)\right| \leq \frac{1}{16}\left|x_{1}-x_{2}\right|+\frac{1}{16}\left|y_{1}-y_{2}\right|$, and $\left(Q_{1}+Q_{3}\right) \ell_{1}+\left(Q_{2}+Q_{4}\right) \ell_{2} \approx$ $0.217861<1$. Thus all the conditions of Theorem 3.1 are satisfied and consequently, its conclusion applies to the problem (3.13).

\section{Conclusions}

In this paper we introduced and solved a new boundary value problem consisting of nonlinear coupled fractional differential equations and nonlocal coupled integro-multistrip-multipoint boundary conditions. Assuming different conditions on the nonlinear functions involved in the given problem, we have presented the criteria ensuring the existence of solutions for the problem at hand by applying Banach contraction mapping principle, Leray-Schauder alternative and Krasnoselskii fixed point theorem. The obtained results are of quite general nature and lead to several interesting special cases (new results) by fixing the values of the parameters involved in the problem appropriately. For example, if we take all $\beta_{i-1}=0, i=2, \ldots, p$ in the results of this paper, we obtain the ones associated with the boundary conditions of the form:

$$
\begin{aligned}
& x^{(\widehat{i})}(0)=0, \int_{0}^{1} x(s) d s=\sum_{j=1}^{q} \gamma_{j} y\left(\rho_{j}\right), \widehat{i}=0,1,2, \ldots, n-2, \\
& y^{(\widehat{j})}(0)=0, \int_{0}^{1} y(s) d s=\sum_{i=2}^{\mu} \beta_{i-1}^{\prime} \int_{\theta_{i-1}}^{\theta_{i}} x(s) d s+\sum_{j=1}^{\lambda} \gamma_{j}^{\prime} x\left(\zeta_{j}\right), \widehat{j}=0,1,2, \ldots, m-2 .
\end{aligned}
$$

Letting all $\beta_{i-1}^{\prime}=0, i=2, \ldots, p$, our results correspond to the ones with the boundary conditions:

$$
\begin{aligned}
& x^{(\widehat{i})}(0)=0, \int_{0}^{1} x(s) d s=\sum_{i=2}^{p} \beta_{i-1} \int_{\eta_{i-1}}^{\eta_{i}} y(s) d s+\sum_{j=1}^{q} \gamma_{j} y\left(\rho_{j}\right), \widehat{i}=0,1,2, \ldots, n-2, \\
& y^{(\widehat{j})}(0)=0, \int_{0}^{1} y(s) d s=\sum_{j=1}^{\lambda} \gamma_{j}^{\prime} x\left(\zeta_{j}\right), \widehat{j}=0,1,2, \ldots, m-2 .
\end{aligned}
$$

In case all $\beta_{i-1}=0, i=2, \ldots, p$ and $\beta_{i-1}^{\prime}=0, i=2, \ldots, \mu$, the results of this paper lead to the ones for the integro-multipoint boundary conditions:

$$
x^{(\widehat{i})}(0)=0, \int_{0}^{1} x(s) d s=\sum_{j=1}^{q} \gamma_{j} y\left(\rho_{j}\right) ; y^{(\widehat{j})}(0)=0, \int_{0}^{1} y(s) d s=\sum_{j=1}^{\lambda} \gamma_{j}^{\prime} x\left(\zeta_{j}\right),
$$

where $\widehat{i}=0,1,2, \ldots, n-2$ and $\widehat{j}=0,1,2, \ldots, m-2$.

Fixing all $\gamma_{j}=0, j=1, \ldots, q$ and $\gamma_{j}^{\prime}=0, j=1, \ldots, \lambda$ in the results of this paper, we get the ones associated with coupled integro-multistrip conditions of the form:

$$
\begin{aligned}
& x^{(\widehat{i})}(0)=0, \int_{0}^{1} x(s) d s=\sum_{i=2}^{p} \beta_{i-1} \int_{\eta_{i-1}}^{\eta_{i}} y(s) d s, \widehat{i}=0,1,2, \ldots, n-2, \\
& y^{(\widehat{j})}(0)=0, \int_{0}^{1} y(s) d s=\sum_{i=2}^{\mu} \beta_{i-1}^{\prime} \int_{\theta_{i-1}}^{\theta_{i}} x(s) d s, \widehat{j}=0,1,2, \ldots, m-2 .
\end{aligned}
$$




\section{REFERENCES}

[1] I. Podlubny, Fractional Differential Equations, Academic Press, San Diego, 1999.

[2] A.A. Kilbas, H.M. Srivastava, J.J. Trujillo, Theory and Applications of Fractional Differential Equations, North-Holland Mathematics Studies, 204, Elsevier Science B.V., Amsterdam, 2006.

[3] K.S. Miller, B. Ross, An Introduction to the Fractional Calculus and Fractional Differential Equations, Wiley and Sons, New York, 1993

[4] J. Sabatier, O.P. Agrawal, J.A.T. Machado (Eds.), Advances in Fractional Calculus: Theoretical Developments and Applications in Physics and Engineering, Springer, Dordrecht, 2007.

[5] S. Liang, J. Zhang, Existence of multiple positive solutions for $m$-point fractional boundary value problems on an infinite interval, Math. Comput. Modelling 54 (2011) 1334-1346.

[6] C. Goodrich, Existence and uniqueness of solutions to a fractional difference equation with nonlocal conditions, Comput. Math. Appl. 61 (2011), 191-202.

[7] J.R. Wang, Y. Zhou, M. Feckan, On recent developments in the theory of boundary value problems for impulsive fractional differential equations, Comput. Math. Appl. 64 (2012), 3008-3020.

[8] B. Ahmad, On nonlocal boundary value problems for nonlinear integro-differential equations of arbitrary fractional order, Results Math. 63 (2013), 183-194.

[9] C. Zhai, L. Xu, Properties of positive solutions to a class of four-point boundary value problem of Caputo fractional differential equations with a parameter, Commun. Nonlinear Sci. Numer. Simul. 19 (2014), 2820-2827.

[10] J. Henderson, N. Kosmatov, Eigenvalue comparison for fractional boundary value problems with the Caputo derivative, Fract. Calc. Appl. Anal. 17 (2014), 872-880.

[11] Y. Ding, Z. Wei, J. Xu, D. O'Regan, Extremal solutions for nonlinear fractional boundary value problems with $p$-Laplacian, J. Comput. Appl. Math. 288 (2015), 151-158.

[12] H. Wang, Existence of solutions for fractional anti-periodic BVP, Results Math. 68 (2015), 227-245.

[13] B. Ahmad, S.K. Ntouyas, Some fractional-order one-dimensional semi-linear problems under nonlocal integral boundary conditions, Rev. R. Acad. Cienc. Exactas Fis. Nat. Ser. A Math. RACSAM 110 (2016), 159-172.

[14] M. Faieghi, S. Kuntanapreeda, H. Delavari, D. Baleanu, LMI-based stabilization of a class of fractional-order chaotic systems, Nonlinear Dynam. 72 (2013), 301-309.

[15] F. Zhang, G. Chen C. Li, J. Kurths, Chaos synchronization in fractional differential systems, Phil. Trans. R. Soc. A 371 (2013), 20120155.

[16] B. Ahmad, S.K. Ntouyas, A. Alsaedi, Fractional differential equations and inclusions with nonlocal generalized RiemannLiouville integral boundary conditions, Int. J. Anal. Appl. 13 (2017), 231-247.

[17] S. Asawasamrit, N. Phuangthong, S.K. Ntouyas, J. Tariboon, Nonlinear sequential Riemann-Liouville and Caputo fractional differential equations with nonlocal and integral boundary conditions, Int. J. Anal. Appl. 17 (2019), 47-63.

[18] C. S. Goodrich, Existence of a positive solution to systems of differential equations of fractional order, Comput. Math. Appl. 62 (2011), 1251-1268.

[19] S.K. Ntouyas, M. Obaid, A coupled system of fractional differential equations with nonlocal integral boundary conditions, Adv. Difference Equ. 2012 (2012), Art. ID 130.

[20] B. Ahmad, S.K. Ntouyas, Existence results for a coupled system of Caputo type sequential fractional differential equations with nonlocal integral boundary conditions, Appl. Math. Comput. 266 (2015), 615-622. 
[21] B. Ahmad, S.K. Ntouyas, A. Alsaedi, On a coupled system of fractional differential equations with coupled nonlocal and integral boundary conditions, Chaos Solitons 83 Fractals 83 (2016), 234-241.

[22] A. Alsaedi, S.K. Ntouyas, D. Garout, B. Ahmad, Coupled fractional-order systems with nonlocal coupled integral and discrete boundary conditions, Bull. Malays. Math. Sci. Soc. 42 (2019), 241-266.

[23] A. Granas, J. Dugundji, Fixed Point Theory, Springer-Verlag, New York, 2005.

[24] M.A. Krasnoselskii, Two remarks on the method of successive approximations, Uspekhi Mat. Nauk 10 (1955), $123-127$. 